\title{
ON POLYNOMIALS WITH LEAST WEIGHTED MAXIMUM
}

\section{F. V. ATKINSON}

1. For the orthogonal polynomials which are associated with a suitably restricted weight-distribution over a real interval it is known [4, Chap. III] that the zeros are all real and simple, and located in the interior of the orthogonality interval; among the numerous further properties is the separation theorem for the zeros of consecutive polynomials of the sequence. The statement concerning the location of the zeros extends to a very wide class of minimal polynomials [2]. The separation property for the zeros of consecutive polynomials (and still more so that for nonconsecutive polynomials) appears on the other hand to be more special. I have shown [1] that this property, for consecutive polynomials, extends to certain polynomials of least mean $p$ th power, for any $p>1$, with continuous weight-function. An extension to polynomials of least mean $p$ th power, with respect to a finite number of points, has been given, together with other results, by Motzkin and Walsh [3].

Here I consider polynomials which are minimal with respect to a weighted uniform norm. This may be regarded [4, pp. 40-41] as the limiting case as $p \rightarrow \infty$ of the mean $p$ th power norm, so that it is to be expected that the separation property will still hold. However, this limiting process would not, without some further refinement, establish the mutual separation of the zeros in the strong sense, and I therefore give here a direct argument, which differs substantially from that for the case of the mean $p$ th power $[1 ; 3]$.

The following is the result to be proved:

ThEOREM. Let $w(x)$ be positive and continuous in the finite real interval $a \leqq x \leqq b$. Let the polynomials $p_{n}(x)(n=1,2, \cdots)$ be of degree $n$, have unit coefficient of $x^{n}$, and minimize the expression

$$
\max _{a \leqq x \leqq b}\left|w(x) p_{n}(x)\right| .
$$

Then between two consecutive zeros of $p_{n}(x)$, for $n \geqq 2$, there lies one zero of $p_{n-1}(x)$.

2. I assemble first known facts regarding the $p_{n}(x)$, with indications of the proofs.

Received by the editors April 18, 1955. 
Minimizing polynomials of (1) exist. We may confine attention to polynomials of the form $\prod_{1}^{n}\left(x-x_{r}\right)$ where all the $x_{r}$ lie in $a \leqq x \leqq b$, since if any of the $x_{r}$ were complex or in the intervals $(-\infty, a)$, $(b, \infty)$ the absolute value of the polynomial could be reduced uniformly in $(a, b)$ by moving the $x_{r}$ in question. This set of polynomials has uniformly bounded coefficients, so that the minimum of (1) is certainly attained for one polynomial $p_{n}(x)$; we denote the minimum of (1) by $k_{n}$.

We next assert that $\left|w(x) p_{n}(x)\right|$ attains the value $k_{n}$ at least $n+1$ times in $a \leqq x \leqq b$. Denoting the $x$-values in question by $y_{1}, \cdots$, $y_{n+1}$, where $a \leqq y_{1}<\cdots<y_{n+1} \leqq b$, we assert further that the $y_{r}$ can be chosen so that $p_{n}\left(y_{r}\right)(r=2, \cdots, n+1)$ has the opposite sign to $p_{n}\left(y_{r-1}\right)$. If this were not the case we could construct a polynomial $q(x)$ of degree less than $n$, which had the same sign as $p_{n}(x)$ for all $x$-values in $a \leqq x \leqq b$ for which $\left|w(x) p_{n}(x)\right|=k_{n}$. It would then follow that for small positive $\epsilon$ the polynomial $p_{n}(x)-\epsilon q(x)$ would have the property that $\left|w(x)\left(p_{n}(x)-\epsilon q(x)\right)\right|<k_{n}$ for $a \leqq x \leqq b$, in contradiction to the definition of $p_{n}(x)$ and $k_{n}$.

The uniqueness now follows. Were there two such polynomials $p_{n}(x), p_{n}^{*}(x)$, consideration of their arithmetic mean would show that the functions $w(x) p_{n}(x), w(x) p_{n}^{*}(x)$ would have to attain together one or other of the values $\pm k_{n}$ for $n+1$ distinct $x$-values in $a \leqq x \leqq b$. Since however $p_{n}(x)$ and $p_{n}{ }^{*}(x)$ are of degree $n$ they cannot agree at $n+1$ points without being identical.

3. The proof of the theorem depends on the following

LEMMA. Let $f(x), g(x)$ be polynomials with real coefficients, and write $F(x, c)=f(x)-c g(x)$. Let $c_{0}, \epsilon$ be fixed real numbers with $\epsilon>0$. Let $F(x, c)$ have exclusively real and simple zeros in $x$ when $c$ is such that either $c_{0}-\epsilon<c<c_{0}$ or $c_{0}<c<c_{0}+\epsilon$. Then $F\left(x, c_{0}\right)$ can have no double zero which is not also a zero of $g(x)$.

We observe first that the zeros of $F\left(x, c_{0}\right)$ must all be real; this follows from the hypotheses and the continuous dependence on $c$ of the zeros of $F(x, c)$, in view of Rouché's theorem, for example. Let us suppose if possible that $F\left(x, c_{0}\right)$ had a real zero of the second (and no higher) order at $x=x_{0}$. We have then

$$
f\left(x_{0}\right)-c_{0} g\left(x_{0}\right)=0, \quad f^{\prime}\left(x_{0}\right)-c_{0} g^{\prime}\left(x_{0}\right)=0,
$$

and also

$$
f^{\prime \prime}\left(x_{0}\right)-c_{0} g^{\prime \prime}\left(x_{0}\right) \neq 0, \quad g\left(x_{0}\right) \neq 0
$$


We then deduce that for some $c$ near to $c_{0}$ the polynomial $F(x, c)$ must have complex zeros, in contradiction to the hypotheses.

Let $c=c_{0}+\eta$, where $\eta$ is real and $0<|\eta|<\epsilon$; write also $x=x_{0}+\xi$. Expanding in powers of $\xi$ as far as terms in $\xi^{2}$ we have, using (2),

$$
\begin{aligned}
F\left(x_{0}+\xi, c_{0}+\eta\right)= & -\eta g\left(x_{0}\right)-\xi \eta g^{\prime}\left(x_{0}\right) \\
& +2^{-1} \xi^{2}\left\{f^{\prime \prime}\left(x_{0}\right)-c_{0} g^{\prime \prime}\left(x_{0}\right)-\eta g^{\prime \prime}\left(x_{0}\right)\right\}+\cdots .
\end{aligned}
$$

Let us denote the $\xi$-values for which $F\left(x_{0}+\xi, c_{0}+\eta\right)=0$ by $\xi_{1}, \xi_{2}, \cdots$. Since $g\left(x_{0}\right) \neq 0$, these are necessarily different from zero, and by the elementary theory of equations we have

$$
\begin{aligned}
\sum \xi_{r}^{-2}= & \left(g^{\prime}\left(x_{0}\right) / g\left(x_{0}\right)\right)^{2} \\
& +\left\{\eta g\left(x_{0}\right)\right\}^{-1}\left\{f^{\prime \prime}\left(x_{0}\right)-c_{0} g^{\prime \prime}\left(x_{0}\right)-\eta g^{\prime \prime}\left(x_{0}\right)\right\} .
\end{aligned}
$$

Now in view of the inequalities (3) the right-hand side of (4) becomes infinite as a multiple of $\eta^{-1}$ for small $\eta$, and so takes different signs for small positive and small negative $\eta$. On the other hand the lefthand side of (4) is necessarily positive, the $\xi_{r}$ being by hypothesis all real. This gives a contradiction, and so proves the lemma.

4. We pass to the proof of the theorem. As in previous investigations $[1 ; 3]$ it is sufficient for this purpose to prove that the polynomial $P_{n}(x, c)=p_{n}(x)-c p_{n-1}(x)$ has, for $n \geqq 2$ and any real $c$, only real and simple zeros.

Take first $c$-values such that $|c|<k_{n} / k_{n-1}$. We assert that in this case $P_{n}(x, c)$ has $n$ simple zeros which lie one in each of the intervals $\left(y_{1}, y_{2}\right), \cdots,\left(y_{n}, y_{n+1}\right)$, where the $y_{r}$ are as in $\$ 2$. We have in fact

$$
\begin{aligned}
\left|w\left(y_{r}\right) p_{n}\left(y_{r}\right)\right| & =k_{n}, \\
\left|c w\left(y_{r}\right) p_{n-1}\left(y_{r}\right)\right| & \leqq\left|c k_{n-1}\right|<k_{n} .
\end{aligned}
$$

This shows that $P_{n}\left(y_{r}, c\right)$ has the same sign as $p_{n}\left(y_{r}\right)$. Since $p_{n}\left(y_{r}\right)$ and $p_{n}\left(y_{r-1}\right)$ have opposite signs for $r=2, \cdots, n+1$, the statement is proved.

A similar argument applies if $|c|>k_{n} / k_{n-1}$. In this case we can assert that $P_{n}(x, c)$ has the same sign as $-c p_{n-1}(x)$ at $x$-values for which $\left|w(x) p_{n-1}(x)\right|=k_{n-1}$. We can assert the existence of $n$ such $x$-values in $a \leqq x \leqq b$, between any two of which $p_{n-1}(x)$ changes sign. Thus $P_{n}(x, c)$ has at least $n-1$ real zeros of odd order, and thus exactly $n$ real simple zeros.

We have thus proved that $P_{n}(x, c)$ has all its zeros real and simple for all real $c$, with the possible exception of $c= \pm k_{n} / k_{n-1}$, and it 
remains to establish the simplicity of the zeros for these $c$-values also. By the lemma we can assert that any multiple zeros of $P_{n}(x$, $\left.\pm k_{n} / k_{n-1}\right)$ must be either zeros of the third or higher order, or else double zeros which are also zeros of $p_{n-1}(x)$. We proceed to eliminate these two possibilities.

Suppose then that $P_{n}\left(x, k_{n} / k_{n-1}\right)$ had a multiple zero $x^{*}$ say. Then for $c$-values near to $k_{n} / k_{n-1}$ there would have to be a corresponding number of zeros of $P_{n}(x, c)$, which tended to $x^{*}$ as $c \rightarrow k_{n} / k_{n-1}$. We have however shown that for $-k_{n} / k_{n-1}<c<k_{n} / k_{n-1}$ the zeros of $P_{n}(x, c)$ are separated by the values $y_{2}, \cdots, y_{n}$ so that as $c \rightarrow k_{n} / k_{n-1}$ from below at most two zeros can tend to any one value $x^{*}$, and then only if $x^{*}$ is one of the values $y_{2}, \cdots, y_{n}$. It follows that $P_{n}(x$, $k_{n} / k_{n-1}$ ) cannot have a zero of higher than the second order, and then only at the values $y_{2}, \cdots, y_{n}$. The same reasoning obviously applies to $P_{n}\left(x,-k_{n} / k_{n-1}\right)$. We have thus reduced the possibilities to double zeros at the points $y_{2}, \cdots, y_{n}$ which are also zeros of $p_{n-1}(x)$. This however is easily seen to be impossible. If $P_{n}\left(x, \pm k_{n} / k_{n-1}\right)=0$ for $x=y_{r}$, say, then

$$
\left|\left(k_{n} / k_{n-1}\right) p_{n-1}\left(y_{r}\right)\right|=\left|p_{n}\left(y_{r}\right)\right|=k_{n} / w\left(y_{r}\right),
$$

showing that $p_{n-1}\left(y_{r}\right) \neq 0$. Thus $P_{n}\left(x, \pm k_{n} / k_{n-1}\right)$ can have no multiple zeros, and the proof of the theorem is complete.

\section{REFERENCES}

1. F. V. Atkinson, Über die Nullstellen gewisser extremaler Polynome, Archiv der Mathematik vol. 3 (1952) pp. 83-86.

2. L. Fejêr, Über die Lage der Nullstellen von Polynomen, die aus Minimumforderungen gewisser Art entspringen, Math. Ann. vol. 85 (1922) pp. 41-48.

3. T. S. Motzkin and J. L. Walsh, Least pth power polynomials on a real finite point set, Trans. Amer. Math. Soc. vol. 78 (1955) pp. 67-81.

4. G. Szegö, Orthogonal Polynomials, New York, 1939.

Canberra University College 